\title{
Induced Cholesteric Liquid Crystalline Polymers
}

\author{
Takashi Mihara, Kazuhiro Nomura, Keisuke Funaki, \\ and Naoyuki KoIDE \\ Department of Chemistry, Faculty of Science, Science University of Tokyo, \\ 1-3 Kagurazaka, Shinjuku-ku, Tokyo 162, Japan
}

(Received July 24, 1996)

\begin{abstract}
A new type of induced cholesteric liquid crystalline thermotropic polymer was prepared by the copolymerization of the achiral mesogenic monomer, $p$-cyanobiphenyl- $p^{\prime}$-methacryloyloxybenzoate or $p$-cyanobiphenyl- $p^{\prime}$ acryloyloxybenzoate with the chiral and non-mesogenic monomer, 2-methyl butyl methacrylate, 2-methyl-1-butyl acrylate, or l-menthyl methacrylate. The mesogenic group is directly attached to the methacrylate- or acrylate-backbone chain without an alkyl spacer. Homopolymers of the achiral and mesogenic monomers exhibited an enantiotropic nematic phase; however, those of the chiral and non-mesogenic monomers themselves did not exhibit any liquid crystalline phases. Copolymers with a combination of achiral mesogenic monomers and chiral non-mesogenic monomers tended to form the so-called induced cholesteric liquid crystalline phase. The type of liquid crystalline phase and transition temperature were found to be different depending upon the fraction of the chiral non-mesogenic comonomer.

KEY WORDS Side Chain Type Liquid Crystalline Polymer / Induced Cholesteric Phase /
\end{abstract}

Cholesteric liquid crystalline polymers can be obtained by combination of a chiral component and a mesogenic unit in the main chain such as low molecular cholesteric liquid crystalline compounds. Strzelecki et al. reported that cholesteric polyesters are obtained by transesterification of a mesogenic monomer with a chiral diacid, (+)-3-methyladipic acid, or with a mixture of an achiral diacid and chiral diacid. ${ }^{1}$ Blumstein et al. studied cholesteric copolymers prepared by azoxydibenzoate mesogenic units and a mixture of decanedioic acid and (+)-3-methyladipic acid. ${ }^{2}$ Similar cholesteric polyesters and copolyesters based on azodibenzoate mesogenic units and (+)-3-methyladipic acid were obtained in our previous work. ${ }^{3}$ Krigbaum et al. synthesized a cholesteric copolymer of 4,4'-dihydroxy- $\alpha$-methylstilbene with (+)methyladipic acid. ${ }^{4}$ The cholesteric phase of the methylstilbene type polyester was found to be maintained below the glass transition temperature upon cooling.

The preparation of the cholesteric polymer by introducing a chiral non-mesogenic moiety to the nematogenic polymer has been studied for main chain type liquid crystalline polymers.

In the case of the side chain type liquid crystalline polymers without a spacer between the polymer backbone and mesogenic pendant group, homopolymer containing a cholesteryl moiety in the mesogenic monomer did not always exhibit a cholesteric mesophase. Finkelmann et al. reported that enantiotropic polymers were obtained by the copolymerization of two cholesteryl methacrylate monomers having different flexible spacer lengths only with equimolar ratios. ${ }^{5}$ Cholesteric polymers were prepared by the copolymerization of a nematogenic monomer and a chiral monomer containing a rigid core. ${ }^{6}$

The present paper deals with a new preparation of a side chain type thermotropic cholesteric polymer with pendant mesogenic groups linked directly to the polymer backbone without flexible spacers. The induced cholesteric polymer was prepared by the copolymerization of an achiral mesogenic monomer, $p$-cyanobiphenyl- $p^{\prime}$ methacryloyloxybenzoate, or $p$-cyanobiphenyl- $p^{\prime}$-acrylo- yloxybenzoate with a chiral non-mesogenic monomer, 2-methyl-1-butyl methacrylate, 2-methyl-1-butyl acrylate, or 1-menthyl methacrylate.

The influence of the polymer backbone structure on the mesomorphic nature is of interest, because the molecular motion of the methacrylate type of polymer backbone would be hindered by the steric effect of $\alpha$-methyl substitution compared with that of the acrylate type of polymer. The difference in the flexibility of the main chain would influence the cholesteric behaviors.

The mixture of the homopolymers of the achiral mesogenic polymer and chiral non-mesogenic polymer did not exhibit a clear cholesteric phase. The cholesteric phase can be produced by the copolymerization of an achiral mesogenic monomer with a chiral non-mesogenic monomer.

\section{RESULTS AND DISCUSSION}

The synthetic routes of a mesogenic monomer with no flexible spacer and chiral monomers are shown in Schemes 1 and 2, respectively. The chiral monomers were in a liquid state at room temperature. Menthol was selected as a chiral compound with high specific rotation.

Only one peak assigned to the melting point for the mesogenic monomer was demonstrated during the heating run for the differential scanning calorimetry (DSC) measurements. A schlieren texture for $p$-cyanobiphenyl- $p^{\prime}$-methacryloylbenzoate (CBMB) was observed above the melting point based on polarizing optical microscopy measurements. This indicates that the CBMB has a nematic phase. A clearing temperature for the $\mathrm{CBMB}$ was not detected with increasing temperature. The schlieren texture of the CBMB was maintained at room temperature during optical polarizing microscopy measurements upon cooling. Thermal polymerization for the CBMB would occur during the heating run. The same results are shown for the CBAB.

The $d$ - and $l$-1-menthyl methacrylates (MtMA*) ex- 
hibited high specific rotations of $+89.8^{\circ}$ and $-90.4^{\circ}$ (c 1.00 tetrahydrofuran (THF) $25^{\circ} \mathrm{C}$ ), respectively, while the 2-methyl-1-butyl methacrylate (MBMA*) showed a specific rotation of $+3.43^{\circ}$, which was very low compared with that of the $d$ - and $l$-MtMA*s.

The structures of the copolymers are shown in Figure 1. The thermal properties for the copolymers were de-

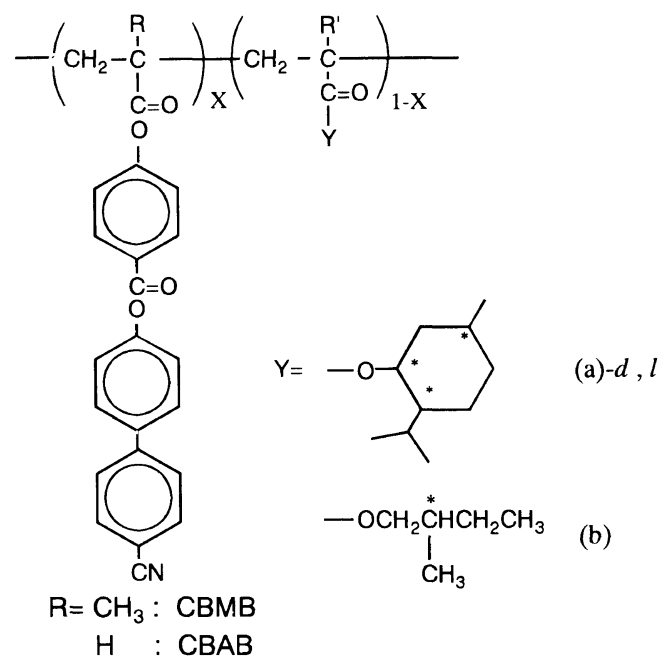

$\mathrm{R}, \mathrm{R}^{\prime}=\mathrm{CH}_{3}, \mathrm{Y}=(\mathrm{a})-d$ : poly(CBMB-co- $\left.d-\mathrm{MtMA}\right)^{*}-x$ $\mathrm{R}, \mathrm{R}^{\prime}=\mathrm{CH}_{3}, \mathrm{Y}=(\mathrm{a})-l$ : poly(CBMB-co-l-MtMA)* $-x$ $\mathrm{R}, \mathrm{R}^{\prime}=\mathrm{CH}_{3}, \mathrm{Y}=(\mathrm{b}):$ poly(CBMB-co-2-MBMA) $*-x$ $\mathrm{R}, \mathrm{R}^{\prime}=\mathrm{H}, \quad \mathrm{Y}=(\mathrm{b}): \operatorname{poly}(\mathrm{CBAB}-\mathrm{co-2}-\mathrm{MBA})^{*}-x$

Figure 1. Structure of side chain type liquid crystalline copolymer. termined using polarizing optical microscopy, DSC and X-ray measurements. Thermal properties, molecular weight and specific rotation for the copolymers are shown in Tables I, II, III, and IV, respectively. Transition temperatures as a function of the fraction of mesogenic units in the copolymers are plotted in Figures 2, 3, 4, and 5 , respectively. The fraction of mesogenic unit for all the copolymers was determined by NMR measurements. The feed fraction of mesogenic monomer for the synthesis of copolymers agreed with the calculated fraction of mesogenic units in the copolymers. We synthesized the copolymer with a $1 \times 10^{4}-2 \times 10^{4}$ number average molecular weight, because it has been reported that the phase transition temperatures remain constant for the liquid crystalline polymer with molecular weights above $1 \times 10^{4} .^{7}$ Specific rotations for all the copolymers increased with decreasing fraction of the mesogenic units. The specific rotation of the chiral homopolymers containing the menthyl groups was larger than that of the chiral monomers, while the specific rotation of the chiral homopolymer with the 2-methyl butyl group was smaller than that of the chiral monomer. The copolymers, poly(CBMB-co- $d$-MtMA)*, poly(CBMB-co-l-MtMA)*, and poly(CBAB-co-2-MBA)*, containing more than $60 \mathrm{~mol} \%$ mesogenic units showed mesomorphic properties. The phase transition temperatures of poly(CBMB-co- $d$-MtMA)* were nearly equal to those of poly(CBMB-co-l-MtMA)*. The phase transition temperatures were independent on the antipode of the menthyl groups in the copolymers. In contrast,

Table I. Phase transition temperatures, molecular weight, and specific rotation of poly (CBMB-co-l-MtMA)*-x

\begin{tabular}{|c|c|c|c|c|c|c|c|}
\hline \multicolumn{2}{|c|}{ Meosgenic monomer fraction $/ \mathrm{mol} \%$} & \multirow{2}{*}{\multicolumn{3}{|c|}{ Phase transition temp $/{ }^{\circ} \mathrm{C}$}} & \multirow{2}{*}{$\bar{M}_{n} / 10^{4}$} & \multirow{2}{*}{$\bar{M}_{w} / \bar{M}_{n}$} & \multirow{2}{*}[\alpha]{$_{\mathrm{D}}^{25}$} \\
\hline Feed & Calcd $^{\mathrm{a}}$ & & & & & & \\
\hline 100 & $-^{\mathrm{b}}$ & $\mathrm{K} \stackrel{181.3}{\longrightarrow} \mathrm{M}$ & 270.9 & $\mathrm{~N} \stackrel{320}{\longrightarrow}$ Decom & $1.27^{\mathrm{c}}$ & 1.36 & $\perp^{\mathrm{d}}$ \\
\hline 90 & $-^{\mathrm{b}}$ & $\mathrm{K} \stackrel{183.2}{\longrightarrow} \mathrm{M}$ & $\stackrel{274.0}{\longrightarrow}$ & $\mathrm{N} \stackrel{307.0}{\longrightarrow}$ Decom & 0.95 & 1.94 & -3.1 \\
\hline 80 & 79.3 & $\mathrm{~K} \stackrel{192.1}{\longrightarrow} \mathrm{M}$ & 265.9 & $\mathrm{Ch} \stackrel{303}{\longrightarrow}$ Decom & 1.41 & 1.40 & -10.5 \\
\hline 70 & 71.5 & $\mathrm{~K} \stackrel{197.5}{\longrightarrow} \mathrm{M}$ & 267.7 & $\mathrm{Ch} \stackrel{307}{\longrightarrow}$ Decom & 1.79 & 1.72 & -18.6 \\
\hline 60 & 60.6 & $\mathrm{~K} \stackrel{195.3}{\longrightarrow} \mathrm{M}$ & 271.2 & $\mathrm{Ch} \stackrel{310}{\longrightarrow}$ Decom & 3.07 & 2.20 & -26.6 \\
\hline 50 & 53.6 & $\mathrm{~K} \rightleftharpoons$ & $\begin{array}{c}166.9 \\
165.9\end{array}$ & $\Longrightarrow \mathrm{I}$ & 2.21 & 2.53 & -33.3 \\
\hline 40 & 42.7 & $\mathrm{~K} \rightleftharpoons$ & $\begin{array}{c}158.2 \\
145.6\end{array}$ & ${ } \mathrm{I}$ & 2.22 & 2.58 & -46.9 \\
\hline 30 & 38.1 & $\mathrm{~K} \rightleftharpoons$ & $\begin{array}{c}159.8 \\
155.1\end{array}$ & $=\mathrm{I}$ & 1.94 & 2.68 & -54.8 \\
\hline 20 & 24.5 & $\mathrm{~K}=$ & $\begin{array}{c}157.6 \\
158.9\end{array}$ & $=\mathrm{I}$ & 1.90 & 2.51 & -69.0 \\
\hline 10 & 13.0 & $\mathrm{~K} \rightleftharpoons$ & $\frac{152.1}{145.8}$ & $=\mathrm{I}$ & 1.84 & 2.31 & -84.9 \\
\hline 0 & 0 & $\mathrm{~g}$ & 127.0 & $-I$ & 0.82 & 2.70 & -97.0 \\
\hline
\end{tabular}

${ }^{\text {a }}$ Calculated by NMR. $\quad{ }^{\mathrm{b}}$ Insoluble in $\mathrm{CDCl}_{3} . \quad{ }^{\mathrm{c}}$ Partially soluble in $\mathrm{CHCl}_{3} .{ }^{\mathrm{d}}$ Insoluble in THF. K, solid; M, mosphase,; Ch, cholesteric; $\mathrm{N}$, nematic; I, isotropic. 
Table II. Phase transition temperatures, molecular weight, and specific rotation of poly(CBMB-co- $d$-MtMA $)^{*}-x$

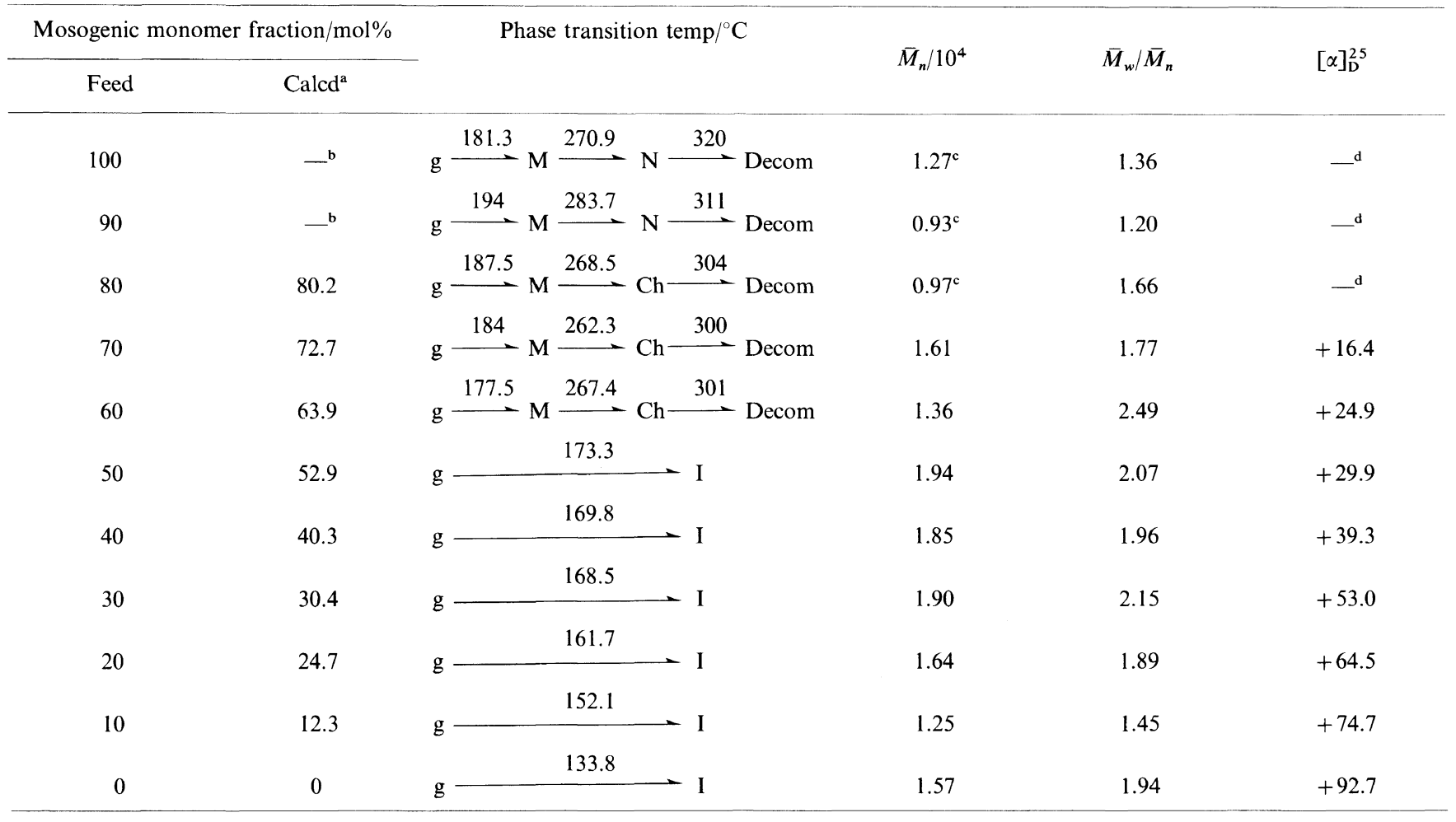

${ }^{a}$ Calculated by NMR. $\quad{ }^{b}$ Insoluble in $\mathrm{CDCl}_{3}$. ${ }^{\mathrm{c}}$ Partially soluble in $\mathrm{CHCl}_{3} . \quad{ }^{\mathrm{d}}$ Insoluble in THF. g, glassy; M, mesophase; Ch, cholesteric; $\mathrm{N}$, nematic; I, isotropic.

Table III. Phase transition temperatures, molecular weight, and specific rotation of poly(CBMB-co-2-MBMA)*-x

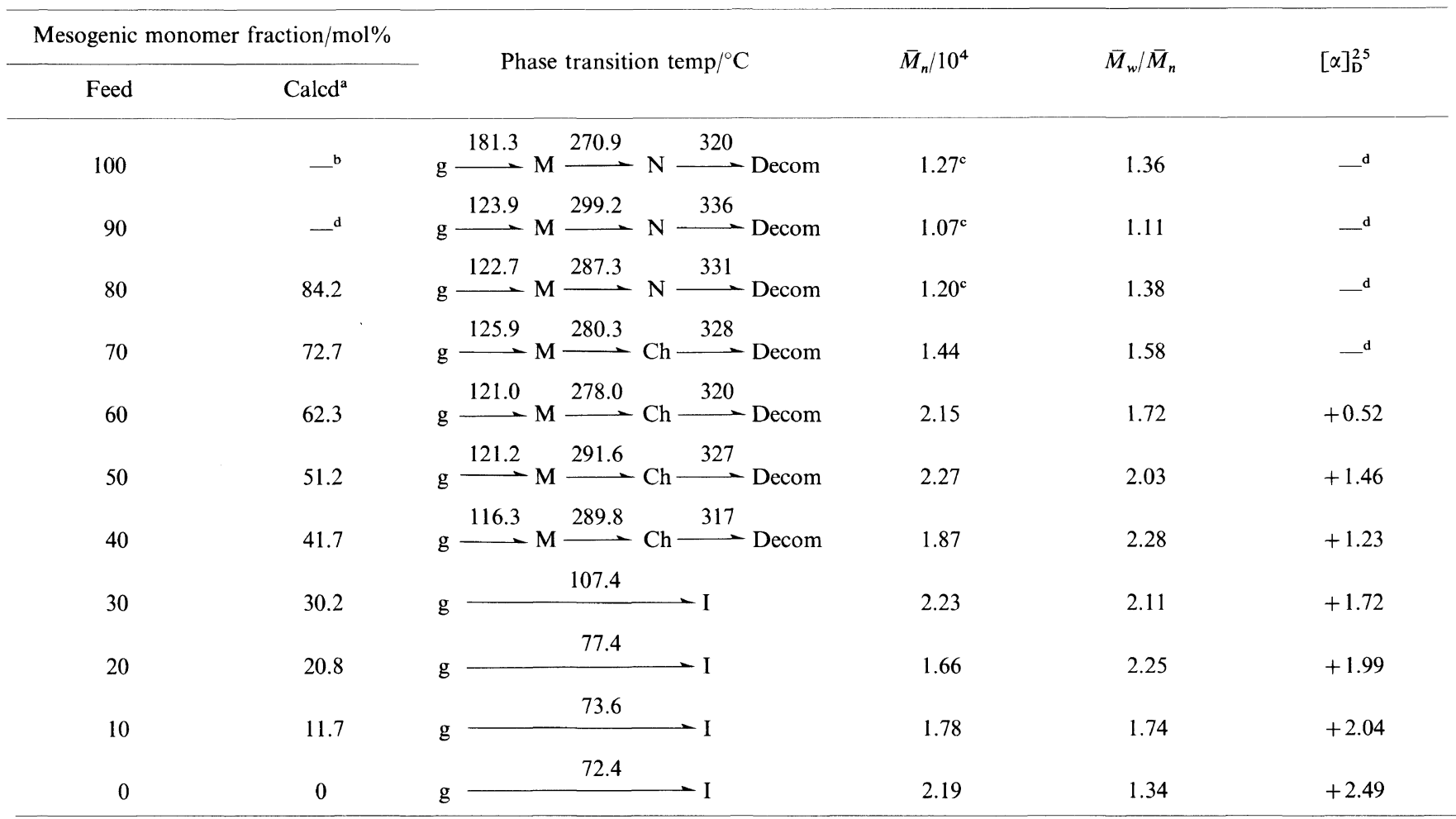

${ }^{a}$ Calculated by NMR. ${ }^{b}$ Insoluble in $\mathrm{CDCl}_{3} . \quad{ }^{\mathrm{c}}$ Partially soluble in $\mathrm{CHCl}_{3} .{ }^{\mathrm{d}}$ Insoluble in THF. g, glassy; M, mesophase; Ch, cholesteric; $\mathrm{N}$, nematic; I, isotropic.

poly(CBMB-co-2-MBMA)* with more than $40 \mathrm{~mol} \%$ mesogenic moieties exhibited mesomorphic properties. The minimal fraction of the mesogenic groups in the copolymer with menthyl groups for exhibiting meso- morphic properties was different from that in the copolymers with 2-methyl butyl groups. The methacrylate copolymers with 2-methyl butyl groups are superior to those with menthyl groups with regard to meso- 
Table IV. Phase transition temperatures and molecular weight, of poly(CBAB-co-2-MBA $)^{*}-x$

\begin{tabular}{ccc}
$\begin{array}{c}\text { Mesogenic monomer } \\
\text { fraction } / \mathrm{mol}^{\mathrm{a}} \mathrm{a}\end{array}$ & $\begin{array}{c}\text { Phase transition } \\
\text { temp } /{ }^{\circ} \mathrm{C}\end{array}$ & $\bar{M}_{n} / 10^{3}$ \\
\hline 100 & $\mathrm{~K} \stackrel{157}{\longrightarrow} \mathrm{N} \stackrel{295}{\longrightarrow} \mathrm{I}$ & 6.3 \\
80 & $\mathrm{~K} \stackrel{139}{\longrightarrow} \mathrm{Ch} \stackrel{250}{\longrightarrow} \mathrm{I}$ & 16.5 \\
71 & $\mathrm{~K} \stackrel{129}{\longrightarrow} \mathrm{Ch} \stackrel{277}{\longrightarrow} \mathrm{I}$ & 15.2 \\
62 & $\mathrm{~K} \stackrel{116}{\longrightarrow} \mathrm{Ch} \stackrel{202}{\longrightarrow} \mathrm{I}$ & 15.7 \\
51 & $\mathrm{~g} \longrightarrow$ & 4.1 \\
\hline
\end{tabular}

${ }^{a}$ Calculated by NMR. g, glassy; K, solid; N, nematic; Ch, cholesteric; I, isotropic.

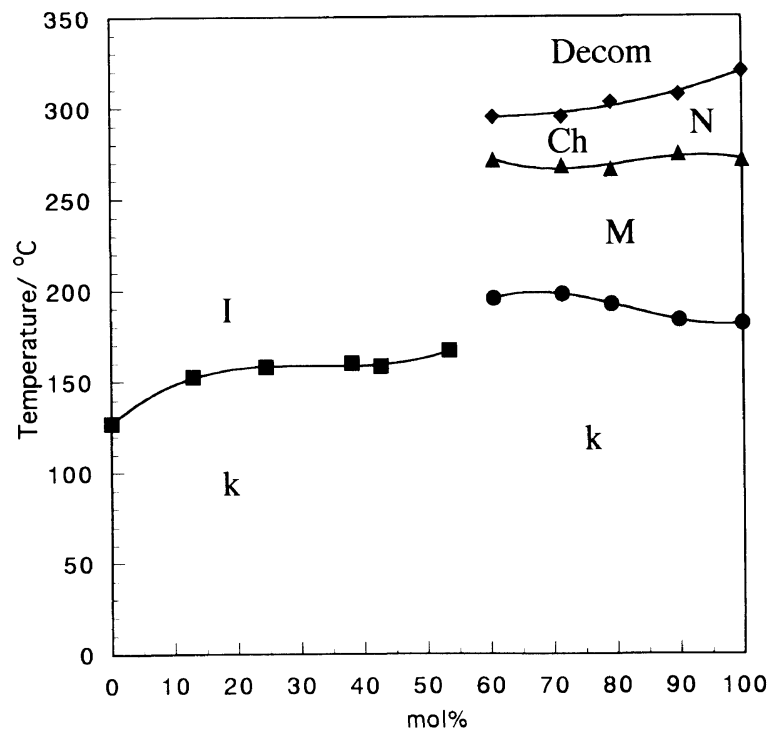

Figure 2. Phase transition temperatures as a function of mesogenic group fraction in poly(CBMB-co-l-MtMA $)^{*}-x$. $\mathrm{k}$, solid; $\mathrm{M}$, mesophase; $\mathrm{Ch}$, cholesteric; $\mathrm{N}$, nematic; $\mathrm{I}$, isotropic.

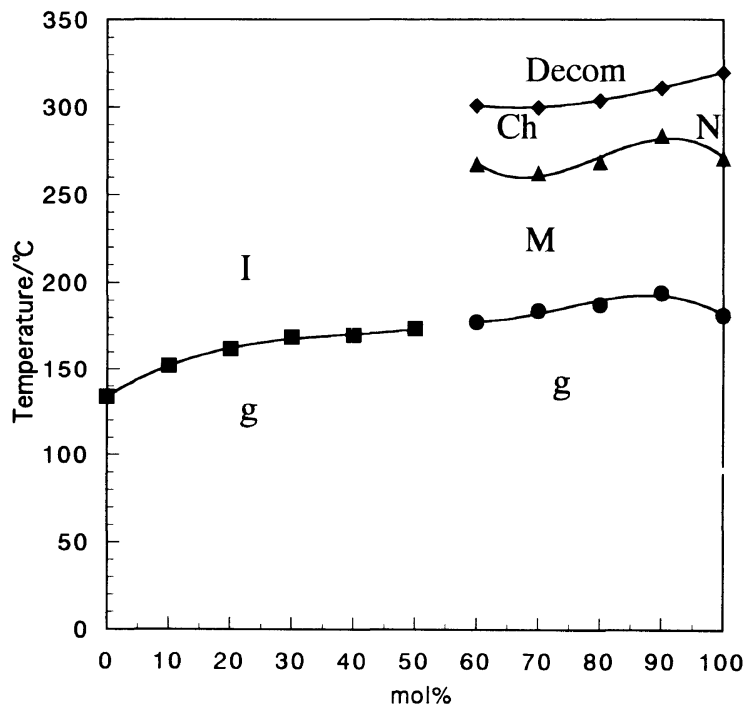

Figure 3. Phase transition temperatures as a function of mesogenic group fraction in poly(CBMB-co- $d$-MtMA)* $-x$. g, glassy; $\mathrm{M}$, mesophase; $\mathrm{Ch}$, cholesteric; $\mathrm{N}$, nematic; $\mathrm{I}$, isotropic.

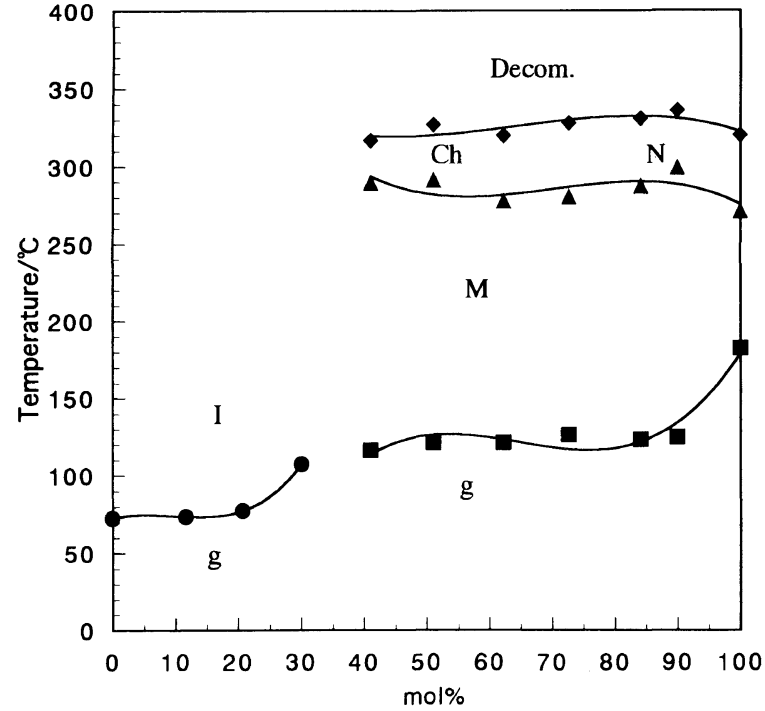

Figure 4. Phase transition temperatures as a function of mesogenic group fraction in poly(CBMB-co-2-MBMA)* $-x$. g, glassy; M, mesophase; $\mathrm{Ch}$, cholesteric; $\mathrm{N}$, nematic, $\mathrm{I}$, isotropic.

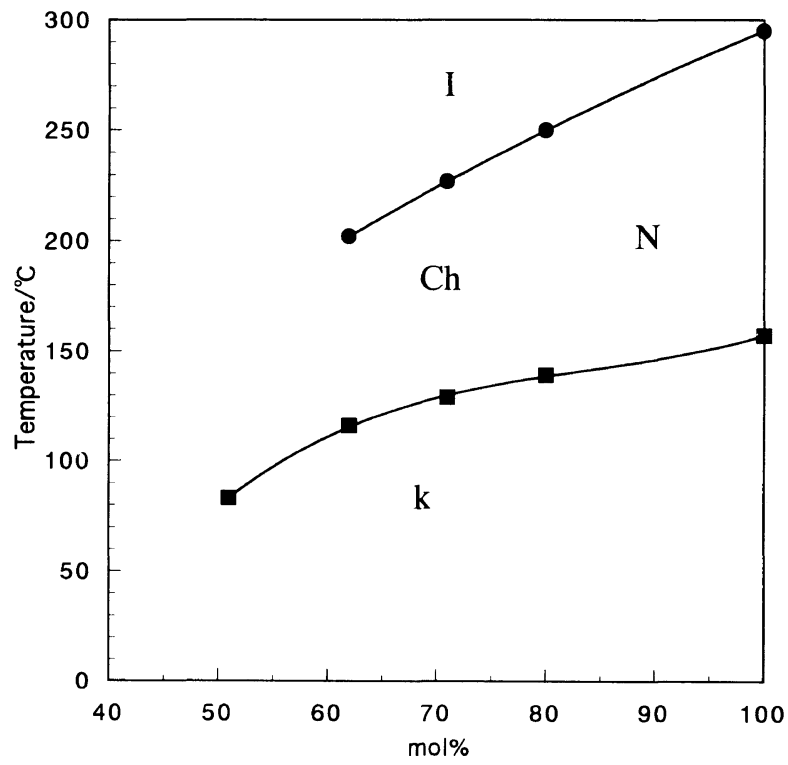

Figure 5. Phase transition temperatures as a function of mesogenic group fraction in poly(CBAB-co-2-MBA)* $-x . \mathrm{k}$, solid; $\mathrm{Ch}$, cholesteric; $\mathrm{N}$, nematic; $\mathrm{I}$, isotropic.

morphic temperature range of the copolymers. The steric factor of the menthol group was bigger than that of the 2-methyl butyl group. The steric factor of the chiral moieties would influence the mesomorphic properties of their copolymers. The menthyl groups would interfere with the alignment of the mesogenic groups which the copolymer contained at the $40-50 \mathrm{~mol} \%$ ratio.

In the case of the copolymers containing polymethacrylate as the polymer backbone, a mesophasenematic phase transition temperature was observed. In the DSC curves for the methacrylate copolymers, two transition temperatures were observed. One was a melting point or glass transition temperature, the other one was a mesophase-cholesteric (nematic) transition temperature. The mesophase observed in the lower mesomorphic temperature range had a higher order in the phase structure than that of the nematic phase which was 
exhibited in the higher temperature range. However, only a broad peak was observed in the X-ray diffraction pattern. Thus, we did not clarify the phase structure of the mesophase. In the X-ray patterns, only one broad peak in the wide angle was observed in both the nematic and cholesteric phases for the poly(CBMB-co$l$-MtMA)* $-x$. Thermal decomposition of the methacrylate copolymers was observed. On the other hand, the copolymer containing polyacrylate as the backbone demonstrated only one mesophase and an isotropic phase. The clearing temperature for the polyacrylate was dependent on the composition of the mesogenic groups in the polyacrylate. A cholesteric or nematic phase was observed for all the copolymers. A cholesteric phase was induced by copolymerization of a nematogenic monomer with a chiral monomer. A cholesteric phase was shown in the methacrylate copolymers containing $40-70 \mathrm{~mol} \%$ or $60-80 \mathrm{~mol} \%$ mesogenic units and in the acrylate copolymers with $60-80 \mathrm{~mol} \%$ mesogenic moieties. A nematic phase was demonstrated in the acrylate and methacrylate copolymers containing a higher fraction of mesogenic groups. The exhibition of an induced cholesteric phase for the copolymers was not dependent on the structure of the chiral moiety.

In the case of two copolymers with 2-methyl butyl groups and a different polymer backbone, poly(CBMBco-2-MBMA)* and poly(CBAB-co-2-MBA)*, the mesomorphic temperature range of the methacrylate copolymer was wider than that of the acrylate copolymer. Furthermore, the methacrylate copolymer exhibited thermal decomposition before displaying the isotropic phase, while the acrylate copolymer showed the clearing temperatures. The difference in the thermal properties was based on the difference in flexibility of the polymer backbone. The methacrylate copolymer was superior to the acrylate copolymer with regard to the exhibition of mesomorphic properties. The mesophases were exhibited in the methacrylate copolymers containing more than $40 \mathrm{~mol} \%$ mesogenic units, while the mesophases were shown in the acrylate copolymers with more than 60 mol\% mesogenic units. However, with regard to the appearance of the induced cholesteric phase temperature range, the acrylate copolymers were superior to the methacrylate copolymers.

For acrylate and methacrylate copolymers with 2-

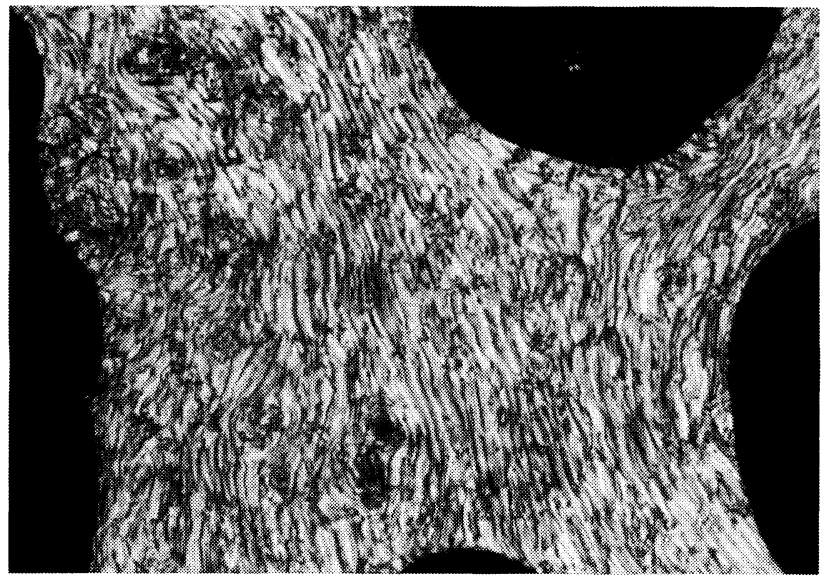

Figure 6. Optical texture of poly(CBMB-co-2-MBMA)*-72.7 at $224.7^{\circ} \mathrm{C}$. methyl butyl groups, clear fingerprint textures were observed in the cholesteric phase, as shown in Figure 6. Helical pitch length for the copolymers with 2-methyl butyl groups decreased with increasing composition of the chiral moiety. The pitch length for the copolymers with 2-methyl butyl groups increased with increasing temperature.

The color of the sample after the cooling run during the optical microscopy measurements for the methacrylate copolymers with menthyl groups was violet, blue, and orange for the copolymers containing 60,70 , and $80 \mathrm{~mol} \%$ mesogenic units, respectively. This coloration of the copolymers originated from the selective reflection of visible light whose wavelength was related to the helical pitch length of the cholesteric phase. The pitch length of the methacrylate copolymers with menthyl groups decreased with increasing composition of the menthyl group. If a cholesteric phase had a longer helical pitch length than the wavelength of visible light, a fingerprint texture or a focal-conic texture was usually observed. In contrast, for the cholesteric phase with the same pitch length as wavelength of visible light, a blue texture for poly(CBMB-co- $d$-MtMA)* -72.7 was observed using orthoscopic observations. However, no selective reflection of the acrylate and methacrylate copolymers with 2-methyl butyl groups was observed. The selective reflection of visible light was dependent on the chemical structure of the chiral moieties.

The specific rotation of poly(CBMB-co-l-MtMA)*71.5 was nearly equal to that of poly(CBMB-co- $d$ MtMA)*-72.7. However, the specific rotation for poly(CBMB-co- $l$-MtMA)* -71.5 and poly(CBMB- $c o-d-$ MtMA)* 72.7 were opposite in sign. We investigated the optical texture for the mixture of poly(CBMB-co-lMtMA)* 71.5 with poly(CBMB-co-d-MtMA)* -72.7 . The polymer mixture exhibited a schlieren texture. The selective reflection of visible light was not observed. This indicates that the polymer mixture did not exhibit a cholesteric phase.

In the case of low molecular weight liquid crystalline mixtures, the so-called induced cholesteric phase usually appeared by adding a chiral compound, which is not necessarily the mesogenic one, to nematogenic materials. In the present investigation, a mixture of a nematic homopolymer, poly(CBMB-co-2-MBMA)*-100 and chiral non-mesogenic homopolymer, poly(CBMB-co-2MBMA)* -0 , in the ratio $70 \mathrm{~mol} \%$ in which the composition of the cholesteric phase appeared in the case of the copolymer, did not reduce typical cholesteric texture, and exhibited only a nematic schlieren texture similar to that observed for poly(CBMB-co-2-MBMA)* containing mesogenic group above $80 \mathrm{~mol} \%$. This suggests that the induced cholesteric polymers are only obtained by the copolymerization of an achiral mesogenic comonomer with a chiral and non-mesogenic monomer, and the content of a chiral non-mesogenic moiety in the copolymer plays an important role in controlling the cholesteric pitches.

\section{CONCLUSIONS}

Induced cholesteric phases were exhibited for copolymers containing more than 40 or $60 \mathrm{~mol} \%$ mesogenic 
groups. The exhibition of the induced cholesteric phase was not dependent on the chemical structures of the chiral moieties. The selective reflection of visible light was observed only for the copolymers with menthyl groups. The mesomorphic temperature range of the methacrylate copolymers with a 2-methyl butyl group was wider than that of the methacrylate copolymers with a menthyl group.

\section{EXPERIMENTAL}

\section{Materials}

The synthesis routes of the mesogenic and chiral monomers are shown in Schemes 1 and 2, respectively. The procedure is described below.

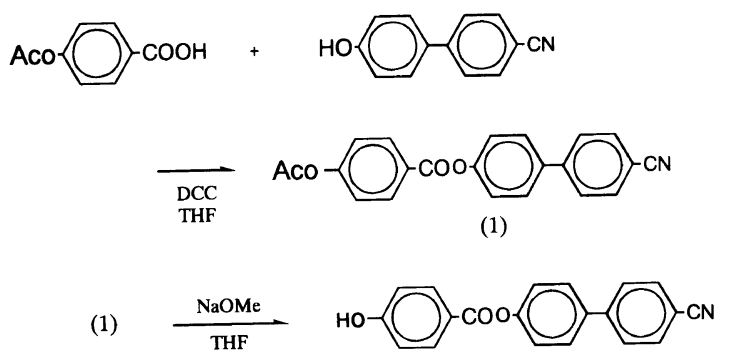

(2)

(2)

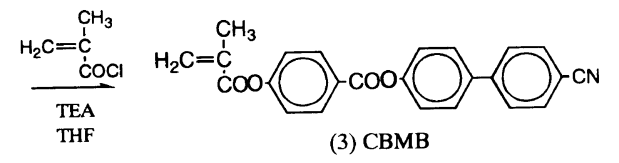

Scheme 1. Synthesis of mesogenic monomer.

(A)

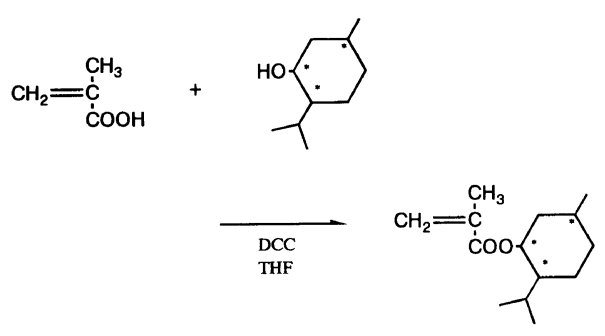

(B)

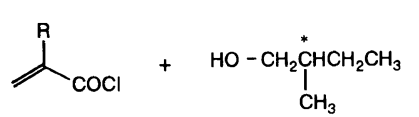

$$
\begin{aligned}
& \overrightarrow{\text { TEA, dry-benzene }}
\end{aligned}
$$

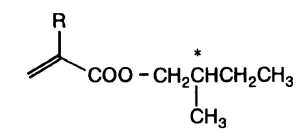

$$
\begin{aligned}
& \text { 2-MBMA* }(\mathrm{R}=\mathrm{CH} 3) \\
& \text { 2-MBA* }(\mathrm{R}=\mathrm{H})
\end{aligned}
$$

Scheme 2. Synthesis of chiral monomers.

Synthesis of Non-Mesogenic Chiral Monomer (lMenthyl Methacrylate). In a dry vessel, a dichloromethane solution of $N, N^{\prime}$-dicyclohexylcarbodiimide $(39.6 \mathrm{~g}, 0.192 \mathrm{~mol})$ was added to a dichloromethane solution of $l$-menthol $(25 \mathrm{~g}, 0.160 \mathrm{~mol})$, methacrylic acid $(16.5 \mathrm{~g}, 0.192 \mathrm{~mol})$, and 4-dimethylaminopyridine $(1.95 \mathrm{~g}$, $\left.1.60 \times 10^{-2} \mathrm{~mol}\right)$. After stirring for 24 hours, the filtrate was concentrated to dryness and purified by column chromatography (eluent chloroform : hexane $=1: 2$ ). This product was obtained in a $52.2 \%$ yield. $[\alpha]_{\mathrm{D}}^{25}=$ $-90.4^{\circ}$ ( $c 1.00$ THF).

NMR $\left(\mathrm{CDCl}_{3}\right) \delta 6.1,5.5\left(\mathrm{~s}, 1 \mathrm{H}, \mathrm{CH}_{2}=\mathrm{C}\right), 4.8(\mathrm{~m}$,

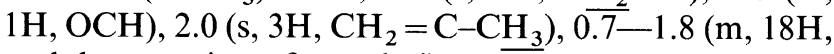
cyclohexane ring of menthol).

IR (neat) $v 1716(\mathrm{COO}), 1639(\mathrm{C}=\mathrm{C})$.

\section{Synthetic Route of Mesogenic Monomer (CBMB)}

4-Cyanobiphenyl-4'-(4-hydroxy)benzoate (2). In an atmosphere of nitrogen, a solution of $N, N^{\prime}$-dicyclohexylcarbodiimide was added to a tetrahydrofuran solution of 4-acetoxybenzoic acid $\left(3.9 \mathrm{~g}, 2.15 \times 10^{-2} \mathrm{~mol}\right)$, 4cyano-4'-hydroxybiphenyl $\left(5.0 \mathrm{~g}, 2.57 \times 10^{-2} \mathrm{~mol}\right)$ and 4-dimethylaminopyridine $\left(0.3 \mathrm{~g}, 2.57 \times 10^{-3} \mathrm{~mol}\right)$. After stirring for 20 hours, the precipitate was removed by filtration. Sodium methylate $(28 \%$ in methanol, $5.0 \mathrm{~g}$, $2.59 \times 10^{-2} \mathrm{~mol}$ ) was added to the concentrated filtrate and the reaction mixture was stirred for 5 minutes. The reaction mixture was then poured into a hydrochloric acid aqueous solution. The resulting white solid was first washed with water and then purified by washing with methanol. The product was obtained in a $70.6 \%$ yield.

IR (Nujol) v $3383(\mathrm{OH}), 2225(\mathrm{CN}), 1699$ (COO), 1605 , 1589, 1511, 1492 (Ar).

\section{4-Cyanobiphenyl-4'-(4-methacryloyloxy)benzoate (3)}

In an atmosphere of nitrogen, a tetrahydrofuran solution of methacryloyl chloride $\left(2.8 \mathrm{ml}, 2.9 \times 10^{-2} \mathrm{~mol}\right)$ was added dropwise to a chilled tetrahydrofuran solution of 4-cyanobiphenyl-4'-(4-hydroxy)phenyl carboxylate (2) $\left(7.0 \mathrm{~g}, 2.23 \times 10^{-2} \mathrm{~mol}\right)$. After stirring for 24 hours, the reaction mixture was concentrated. The residue was poured into water and the resulting precipitate was washed first with water, and then purified by washing with methanol. The product was obtained in a $48.2 \%$ yield.

NMR $\left(\mathrm{CDCl}_{3}\right) \delta 7.3-8.4(\mathrm{~m}, 12 \mathrm{H}, \mathrm{Ar}-\underline{\mathrm{H}}), 5.9,7.5(\mathrm{~s}$, $\left.1 \mathrm{H}, \mathrm{CH}_{2}=\mathrm{C}\right), 2.1\left(\mathrm{~s}, 3 \mathrm{H}, \mathrm{CH}_{2}=\mathrm{C}-\mathrm{CH}_{3}\right)$.

IR (Nujol) v $2229(\mathrm{CN}), 1733(\mathrm{COO}), 1637(\mathrm{C}=\mathrm{C})$, 1603, 1506 (Ar).

Polymerization. Copolymers were synthesized by copolymerization of the mesogenic monomer with no flexible spacer and the chiral monomer in sealed ampoules with $5.0 \mathrm{~mol} \% \alpha, \alpha^{\prime}$-azobisisobutyronitrile in anhydrous $N, N^{\prime}$-dimethylformamide at $60^{\circ} \mathrm{C}$ for 10 hours. They were also obtained by reprecipitation with methanol. And then dissolved in tetrahydrofuran, reprecipitated with methanol and dried at $40^{\circ} \mathrm{C}$ under vacuum.

\section{Characterization}

${ }^{1} \mathrm{H}-\mathrm{NMR}$ was carried out with a JEOL JPN-PMX60 spectrometer using $\mathrm{CDCl}_{3}$ as the solvent. Infrared spectra were recorded on a JEOL JIR-100 spectrometer. Spectra were collected at $4 \mathrm{~cm}^{-1}$ resolution. DSC measurements were conducted with a Mettler 3000 series. Optical microscopy was performed on a Nikon polarizing optical microscopy equipped with a Mettler FP80 controller and a FP82 hot stage. Gel permeation chromatography (GPC) was carried out with a Tosoh HLC-8020 instrument using chloroform as the eluent, equipped with four columns (TSK gel G4000H $\mathrm{H}_{\mathrm{HR}}, \mathrm{G} 3000 \mathrm{H}_{\mathrm{HR}}, \mathrm{G} 2000 \mathrm{H}_{\mathrm{HR}}$, and $\mathrm{G} 2000 \mathrm{H}_{\mathrm{HR}}$ ). The instrument was calibrated with a 
polystyrene standard. Optical rotation was carried out with JASCO DIP-370 digital polarimeter using tetrahydrofuran as the solvent. X-Ray diffraction patterns were recorded with a Rigaku X-Ray diffractometer RAD2B system with $\mathrm{Ni}$-filtered $\mathrm{Cu}-K_{\alpha}$ radiation.

\section{REFERENCES}

1. D. van Luyen, L. Liebert, and L. Strzelecki, Eur. Polym. J., 16, 307 (1980).
2. A. Blumstein and S.Vilasagar, Mol. Cryst. Liq. Cryst. Lett., 72 , 1(1981).

3. K. Iimura and N. Koide, Rep. Prog. Polym. Phys. Jpn., 25, 297 (1982).

4. W. R. Krigbaum, Mol. Cryst. Liq. Cryst., 78, 79 (1981).

5. H. Finkelmann, H. Ringsdorf, W. Soil, and J. H. Wendorff, Makromol. Chem., 179, 829 (1978).

6. H. Finkelmann, J. Koldehoff, and H. Ringsdorf, Angew. Chem. Int. Ed. Engl., 17 (12), 935 (1978).

7. A. Blumstein, S. Vilasagar, S.Ponrathnam, S. B. Clough, and R. B. Blumstein, J. Polm. Sci., Polm. Phys. Ed., 20, 877 (1982). 